\title{
MOOCs: aproximaciones a una estrategia de divulgación para la apropiación social del conocimiento
}

MOOCs: approximations to a dissemination strategy for the social appropriation of knowledge.

\section{RESUMEN}

La propuesta Educativa de los MOOC de la Pontificia Universidad Javeriana - Cali se basa en un Modelo educativo para proyectos de formación virtual, el cual se fundamenta en los siguientes pilares: La identidad de la educación de la compañía de Jesús, la Pedagogía Ignaciana como un elemento distintivo del Proyecto Educativo Institucional y la perspectiva del uso reflexivo de las TIC en los procesos de enseñanza y aprendizaje. Esta fundamentación ha sido esencial para promover la participación de diversos públicos interesados en el acceso de contenidos relacionados con diversas áreas de conocimiento, con didácticas que rompen con la tradición expositiva y posibilitan hacer accesible, adecuado y significativo, el saber experto por medio del reconocimiento, uso y transformación de las TIC como recursos mediadores que transcienden así la gestión de contenidos para generar aprendizajes significativos y oportunidades para la Universidad de divulgación para la apropiación social del conocimiento.

PALABRAS ClAVE: MOOC, cursos masivos, Aprendizaje Significativo, Educación Virtual, Modelo Educativo.

\begin{abstract}
The educational proposal of the MOOC of the Pontificia Universidad Javeriana is based on an educational model for virtual training projects, which is based on the following pillars: The identity of the education of the company of Jesus, Ignatian Pedagogy as a distinctive element of the Institutional Educational Project and the perspective of the reflective use of ICT in teaching and learning processes. This fundamental basis has been essential to promote participation. The results are related to the areas of knowledge, the didactic activities, the expositive experience and the possibility of making accessible, adequate and significant, the expert saber through recognition. use and transformation of ICT as mediating resources that transcend
\end{abstract}

${ }^{1}$ Master en aplicaciones multimedia. Coordinadora de producción de recursos educativos. Colombia. Adrianamorales@javerianacali.edu.co

${ }^{2}$ Master en E-learning. Directora del Centro de Recursos para el Aprendizaje y la Investigación - CRAI. Colombia. tvalencia@javerianacali.edu.co.

${ }^{3}$ Magister en Tecnología Educativa. Jefe de Javevirtual. Colombia. donovan.delvalle@javerianacali.edu.co. 
as well as content management to generate significant learning and opportunities for the University to disseminate for the social appropriation of knowledge.

\section{KEYWORDS:}

MOOC, mass courses, Significant Learning, Virtual Education, Educational Model.

\section{INTRODUCCIÓN}

En este artículo se describen avances en la experiencia de los MOOCs diseñados y ofertados por la Pontificia Universidad Javeriana, seccional Cali, a partir del Modelo Educativo Virtual. Esta experiencia fue inicialmente presentada en el XXIII Congreso Internacional sobre Educación Bimodal TELEDU 2017, en el cual se socializó la apuesta del Modelo Educativo Virtual, de forma puntual en la generación de estos cursos para trascender la gestión de contenidos hacia el aprendizaje significativo. En esta segunda entrega, se presentan los avances alcanzados, en términos de desafíos, retos y aprendizajes, y la proyección futura de la Universidad en este campo. En el primer apartado se resume el modelo educativo virtual que respalda a los MOOCs de la Pontificia Universidad Javeriana Cali, y se aborda la conceptualización de apropiación social del conocimiento. Después se abordan diversas dimensiones relacionadas con los resultados obtenidos con estos cursos, importantes para la proyección de las propuestas masivas. Finalmente se plantea la proyección al futuro, aprendizajes reflejados en reflexiones frente a las demandas e inversión que requiere la producción de este tipo de cursos, y la apuesta por la apropiación social de conocimiento. El objetivo del documento es dar a conocer los avances de este proyecto y compartir con la comunidad académica nacional prospectivas en torno al tema.

\section{Descripción del Problema}

Los MOOCs son cursos abiertos, en línea y masivos (Massive Open Online Courses, comolo indican sus siglas en inglés), y son una apuesta de la Pontificia Universidad Javeriana - Colombia por contribuir con la calidad en la educación. De forma gratuita se comparte el conocimiento de nuestros expertos en diferentes áreas del conocimiento, derivados de experiencias y procesos de investigación. Es una de las acciones de la Universidad que hacen parte de su responsabilidad social con su entorno local, regional, nacional e internacional. Desde el 2015, la Pontificia Universidad Javeriana hace parte de la plataforma edX, fundada por Massachusetts
Institute of Technology (MIT) y por Harvard University, lo que es un reconocimiento a la calidad ofrecida por la Universidad tanto a nivel de investigación como de docencia, y la posibilidad de visibilizarse a nivel global, al lado de las más reconocidas universidades del mundo.

La aparición de este tipo de cursos supone unos de los mayores fenómenos que ha confrontado a la educación superior en las últimas décadas. (Capdevila \& Aranzadi, 2014). Por esta razón fue importante para la Pontificia Universidad Javeriana de Cali incursionar en esta tendencia, reconociendo su potencial para promover e incentivar estrategias de apropiación social del conocimiento, desde los fundamentos que caracterizan su Proyecto Educativo Institucional (PEI), y su valor a la luz de lo que podía llegar a ser, de sus potencialidades alrededor del "carácter flexible y abierto del aprendizaje que preconizan" (Yuan \& Powell, 2013 citado por Torres \& Gago, 2014: 1).

La apropiación social del conocimiento es la comprensión y asimilación de los conocimientos obtenidos en los procesos de investigación, por parte de los diferentes actores de la sociedad, a través de estrategias encaminadas a socializar estos conocimientos con el objetivo de generar interés en su desarrollo y aplicación; estas estrategias se enmarcan en la sociedad del conocimiento, en nuevas formas de organización social, que demandan acciones amplificadoras e innovadoras, que renueven $y$ difundan el conocimiento $y$ posibiliten la generación de nuevos a partir de la adaptabilidad constante de los individuos, en un contexto de democratización y participación (Marín Agudelo, 2012; Borda \& Maldonado, 2010). Así pues, las estrategias de apropiación social se concentran en dos frentes: en los usos sociales y comerciales de los conocimientos en términos de innovación y por otra, en la promoción de valores favorables concentrados principalmente en estrategias de ilustración en la cultura científica; las cuales se mueven en tres niveles: interesarse/ comprender, validar y utilizar.

En ese sentido, los MOOC se reconocen como una estrategia para lograr dicha apropiación, en la medida en que sus caracteristicas apuntan a 
democratizar el conocimiento y a posibilitar a cualquier persona a acercarse a él por ser: abiertos, por tanto no tienen condiciones para restringir su consumo; sin costo; en línea, lo que desdibuja los límites de tiempo y espacio para acceder a la formación; masivos, puesto que no existe un límite de personas y permite la interacción con personas de todo el mundo; y finalmente, de calidad, puesto que las universidades que los elaboran proveen garantía pues su reputación esta en juego.

Por ello, el diseño de MOOCs de la Universidad se realiza bajo su Modelo Educativo Virtual, que se fundamenta en tres pilares: 1) los lineamientos institucionales, 2) la concepción de la enseñanza y el aprendizaje desde la Pedagogía Ignaciana, como un elemento fundamental y distintivo del PEI y 3) los referentes teóricos, metodológicos y conceptuales que sustentan el uso reflexivo de las TIC en los procesos de enseñanza y aprendizaje. La sinergia de estos tres pilares busca garantizar el desarrollo de competencias en y entre los actores principales del proceso de enseñanza y aprendizaje virtual a favor de la Misión y Visión que la Universidad se ha propuesto (Pontificia Universidad Javeriana Cali, 2010).

Bajo estas premisas, la Universidad se cuestionó frente a ¿Cómo lograr el interés y consumo masivo de contenidos originados de experiencias y procesos de investigación en la comunidad académica interna y externa?

\section{Metodología.}

Esta ponencia se ha abordado a partir de un análisis experimental de las experiencias de cada curso, a través de un estudio descriptivo, analizando datos de la plataforma, desde el año 2016 al 2018. Dentro del marco de este ejercicio, se recogen algunos datos de la experiencia, que provienen de informes de gestión y desempeño que se realiza por cada curso, para garantizar la sistematización de la práctica educativa y procesos de retroalimentación al profesor, así como la evaluación de los resultados de los MOOC y su impacto social, en procesos relacionados a continuación:

- Evaluación del proceso de aprendizaje, por parte de los estudiantes, de forma cuantitativa (a través de encuestas) o cualitativa (a través de foros, análisis de los productos, opiniones de los participantes), dependiendo de la naturaleza del curso, para identificar que se haya logrado tanto el objetivo de aprendizaje como la calidad de contenidos y actividades para conseguirlo.
Bajo estos resultados, se establece el plan de mejoramiento de la siguiente cohorte del MOOC.

- Cantidad de inscritos vs cantidad de certificados y de personas que terminan el curso, teniendo en cuenta que existen diversos perfiles de personas que ingresan a los MOOC.

- Resultados derivados en vinculación del personal de los MOOC en otras ofertas de la Universidad.

\section{Resultados}

Uno de los aspectos más significativos de los MOOC es su capacidad de cobertura para la divulgación del conocimiento, como una estrategia que posibilite la apropiación del conocimiento en diversos escenarios. A la fecha de elaboración de este documento, 120.853 personas han participado en alguno de los cursos de la seccional en Cali, y un total de 1377 han sido certificados. Esta certificación la posibilita edX a través de su plataforma, a partir de un pago y de un proceso de validación de identidad con el respectivo documento valido en cada país.

Frente a estos resultados, se han identificado que la población que consume los MOOC es diversa en cuanto a sus intereses, caracterizados de la siguiente manera:

- Consumidor de contenidos pasivo: Son personas que se inscriben al curso para descargar o visualizar los contenidos, y los almacenan para su utilización presente o futura. Estas personas suelen des inscribirse del curso o permanecer, pero nunca participan.

- Estudiantes activos sin interés de certificación: Personas con interés en la temática, pero que no requieren certificación o título. Permanecen en la dinámica del curso, pero tienen un riesgo de deserción relacionado con la libertad de poder abandonar en cualquier momento.

- Estudiantes activos con interés de obtener certificación: Son los participantes con mayor motivación, pues están interesados en recibir mayor reconocimiento por parte del tutor y de los pares, enfocados en obtener la certificación por fines laborales o personales. Este público es el más involucrado con las ofertas complementarias al MOOC, pues ven en ellas la posibilidad de dar continuidad al crecimiento que están esperando. 


\begin{tabular}{|c|c|c|c|c|c|c|}
\hline & Año & $\begin{array}{l}\text { Versión } \\
\text { Curso }\end{array}$ & Nombre del curso & $\begin{array}{l}\text { Inscripción a } \\
\text { los cursos }\end{array}$ & $\begin{array}{l}\text { Inscripción } \\
\text { verificada }\end{array}$ & $\begin{array}{l}\text { Estudiantes } \\
\text { Aprobados }\end{array}$ \\
\hline 1 & 2016 & 1 & $\begin{array}{l}\text { Adherencia al tratamiento de pacientes con } \\
\text { enfermedad crónica }\end{array}$ & 3341 & 24 & 77 \\
\hline 2 & 2016 & 1 & $\begin{array}{l}\text { Antropología de la Música: El caso de las Músicas } \\
\text { del Pacifico Colombiano }\end{array}$ & 2491 & 1 & 3 \\
\hline 3 & 2016 & 1 & $\begin{array}{l}\text { Aproximación Neuropsicológica de la Lecto- } \\
\text { Escritura en la etapa escolar }\end{array}$ & 4472 & 33 & 114 \\
\hline 4 & 2016 & 1 & Impacto de las TIC en la vida de las familias & 1197 & 13 & 19 \\
\hline 5 & 2017 & 1 & $\begin{array}{l}\text { Diálogo Intercultural para la Gestión de Conflictos } \\
\text { Territoriales }\end{array}$ & 5339 & 57 & 81 \\
\hline 6 & 2017 & 1 & Escribir para Convencer & 45775 & 133 & 769 \\
\hline 7 & 2017 & 2 & Impacto de las TIC en la vida de las familias & 1742 & 13 & 23 \\
\hline 8 & 2017 & 1 & Impacto de la Investigación de Mercados & 6030 & 43 & 216 \\
\hline 9 & 2017 & 2 & $\begin{array}{l}\text { Aproximación Neuropsicológica de la Lecto- } \\
\text { Escritura en la etapa escolar }\end{array}$ & 3974 & 56 & 116 \\
\hline 10 & 2017 & 1 & Lo que todos deberían saber del Genoma Humano & 2876 & 9 & 74 \\
\hline 11 & 2017 & 1 & $\begin{array}{l}\text { Gestión de Riesgos para la sostenibilidad } \\
\text { organizacional }\end{array}$ & 4047 & 74 & 177 \\
\hline 12 & 2017 & 1 & $\begin{array}{l}\text { Seguridad y Salud en el Trabajo: Un derecho } \\
\text { fundamental }\end{array}$ & 5722 & 37 & 266 \\
\hline 13 & 2017 & 2 & $\begin{array}{l}\text { Adherencia al tratamiento de pacientes con } \\
\text { enfermedad crónica }\end{array}$ & 2677 & 26 & 74 \\
\hline 14 & 2017 & 3 & $\begin{array}{l}\text { Aproximación Neuropsicológica de la Lecto- } \\
\text { Escritura en la etapa escolar }\end{array}$ & 3864 & 66 & 120 \\
\hline 15 & 2017 & 1 & Lánzate a la Innovación con Design Thinking & 8900 & 141 & 396 \\
\hline 16 & 2018 & 1 & Sexualidad sin tabú: hablemos con adolescentes & 3161 & 31 & 281 \\
\hline 17 & 2018 & 4 & $\begin{array}{l}\text { Aproximación Neuropsicológica de la Lecto- } \\
\text { Escritura en la etapa escolar }\end{array}$ & 2830 & 53 & 86 \\
\hline 18 & 2018 & 3 & Impacto de las TIC en la vida de las familias & 466 & 5 & 31 \\
\hline 19 & 2018 & 1 & Learning Spanish in Paradise & 4385 & 14 & 215 \\
\hline 20 & 2018 & 2 & Lánzate a la Innovación con Design Thinking & 4954 & 504 & 578 \\
\hline 21 & 2018 & 1 & Perdón y reconciliación: cómo sanar heridas & 2610 & 44 & 83 \\
\hline
\end{tabular}

Cuadro 1. Relación de personas inscritas, personas certificadas y personas que aprobaron el curso

Asimismo, se ha reconocido que las áreas de mayor interés del público participante tienen que ver con la certificación de competencias técnicas, formación para el trabajo y expectativas de mejora laboral en las respectivas empresas donde trabajan, o para su vinculación a una oferta laboral futura. Así pues, en función de la apropiación social del conocimiento, la idea de hacer cursos masivos permite, por su naturaleza, el acercamiento de personas de diversas regiones del mundo (más de 91 países en nuestro caso), en los cuales diversos públicos están accediendo y utilizando los recursos educativos, y aprovechando las aulas virtuales como escenarios para la construcción de comunidades de aprendizaje diversas, dando tanto usos sociales como usos comerciales de los conocimientos. Como consecuencia, se ha aumentado significativamente el reconocimiento de los profesores-tutores a nivel local, regional y nacional, tanto en reconocimiento académico como en aumento de la visibilidad de su investigación, nuevas posibilidades de colaboración con otras instituciones e intervenciones a nivel de consultoría.

\subsection{Frente a la propuesta pedagógica}

Cada uno de los MOOC que se propone se fundamenta en los 5 momentos del Paradigma Pedagógico Ignaciano (Contexto, Experiencia, Reflexión, Acción y Evaluación), están diseñados para lograr un aprendizaje con calidad, que se ve reflejado en: a) la pertinencia de las actividades y recursos propuestos en los momentos de la ruta de aprendizaje, b) el alcance del objetivo de 
aprendizaje del MOOC, c) la coherencia de la propuesta pedagógica y d) la calidad pedagógica del MOOC. Estos principios son ampliamente reconocidos por los estudiantes, y durante cada semana de implementación, los experimentan de forma altamente estructurada (Compañía de Jesús, 2003; Velazco, P. C., 2013). A continuación, la siguiente imagen contiene la descripción base presentada a los estudiantes, correspondiente a los momentos de la ruta:

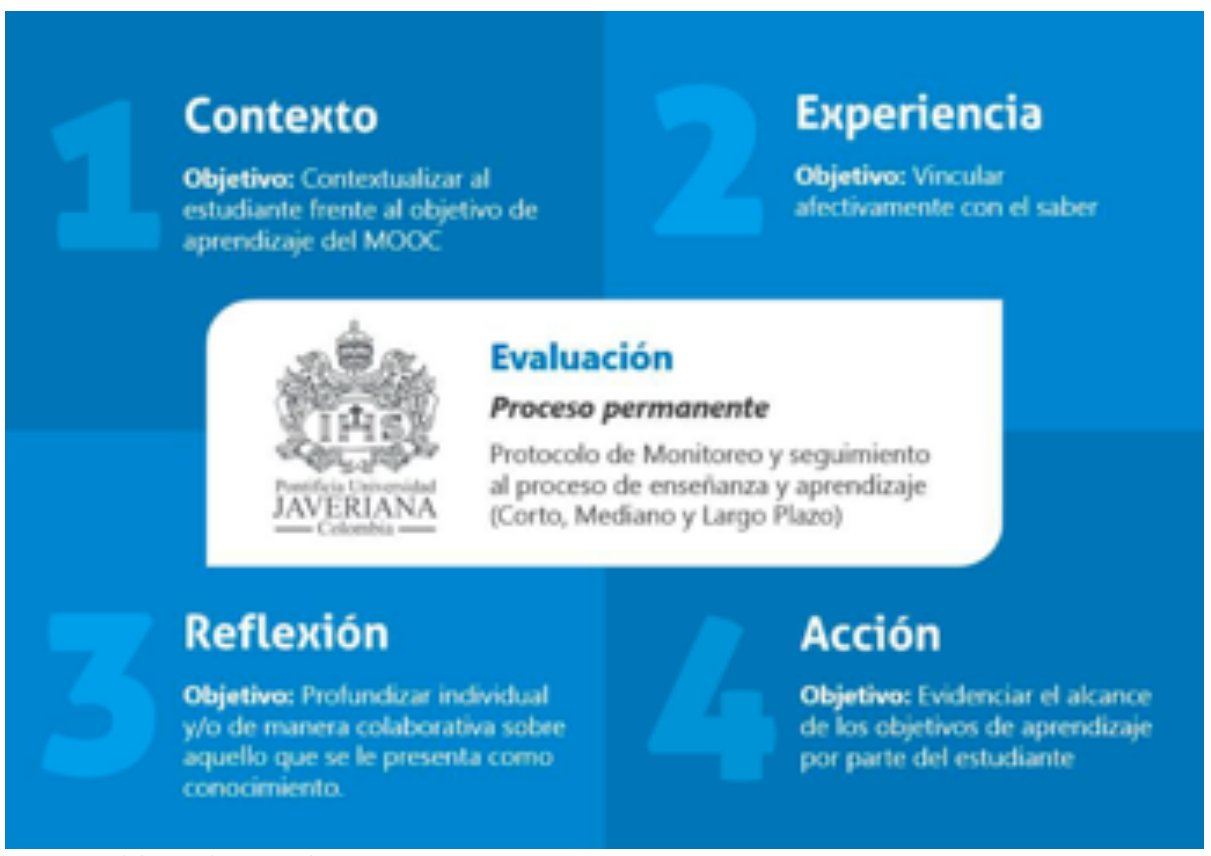

Imagen 1. Los 5 momentos del Paradigma Pedagógico Ignaciano

Frente a este recorrido, los estudiantes tienen la oportunidad de manifestar los aspectos más importantes de su experiencia, en cada una de las estrategias de recolección de información. A continuación, se presentan algunas opiniones sobre las vivencias en algunos de nuestros MOOC

Opinión estudiante 1 2016: "Mi experiencia en el MOOC es satisfactoria, he podido reforzar conocimientos, me pareció muy buena la metodología empleada llevando el tema de la teoría a un caso práctico donde quedaron mucho más claro los componentes teóricos... Muchas gracias y estaré siguiendo la oferta educativa de la Universidad".

Opinion estudante 2, 2017: "Ha sido enriquecedor la experiencia le ha aportado mucho a mi quehacer.... soy docente de niños y niñas ciegos y créanme que me ha despejado cualquier cantidad de dudas que tenía y ha aportado muchos aprendizajes para seguir enseñando a mis estudiantes ciegos desde esta maravillosa postura".

Opinión Estudiante 3, 2016: "Felicidades a las personas encargadas del diseño de este curso de gran calidad. Excelente metodología de enseñanza-aprendizaje, contenido que denota un trabajo investigativo extenso. Valoro con $5 / 5$ el diseño de los recursos audiovisuales y las estrategias de evaluación que me permitieron integrar el conocimiento adquirido con la práctica a partir de los casos".

A nivel general, es ampliamente reconocido por los estudiantes que la propuesta pedagógica es una fortaleza de nuestros cursos y un diferencial. Más del $80 \%$ de los participantes activos la evalúan positivamente (entre buena y excelente) y consideran que contribuye en alto grado para la consecución de los objetivos de aprendizaje.

\subsection{Frente a la identidad gráfica de cada curso y la propuesta audiovisual}

Otro de los aspectos reconocidos por nuestros estudiantes como un diferencial, es la propuesta audiovisual de cada curso. Uno de los esfuerzos significativos que se realiza es proveer de identidad propia a cada MOOC, articulado con la naturaleza didáctica del conocimiento, de tal forma que se relaciona la forma natural y significativa de representar el conocimiento que se quiere enseñar con diversas estrategias didácticas, se proponen alternativas para que el conocimiento del experto 
sea accesible al estudiante, buscando garantizar un aprendizaje significativo a través de poder hacer evidente la estrategia didáctica en el material audiovisual, que es un recurso característico y neurológico de los MOOCs. A continuación, se presenta la identidad de algunos de los cursos La definición de una identidad visual para un curso tiene como fin brindar al usuario (estudiante) una representación gráfica de un tema específico, que además de dar una apariencia atractiva, funciona como guía a través la estructura temática, con el apoyo de diferentes elementos que componen la interfaz de la plataforma educativa. A través de un buen diseño de interfaz, es posible conseguir que la tecnología se convierta en un elemento facilitador de las actividades a realizar. Con el diseño de cada curso, se pretende generar una percepción positiva, no solo en aspectos gráficos, de usabilidad, accesibilidad sino relativos a las emociones, lo cual se traduce en una buena experiencia de usuario.

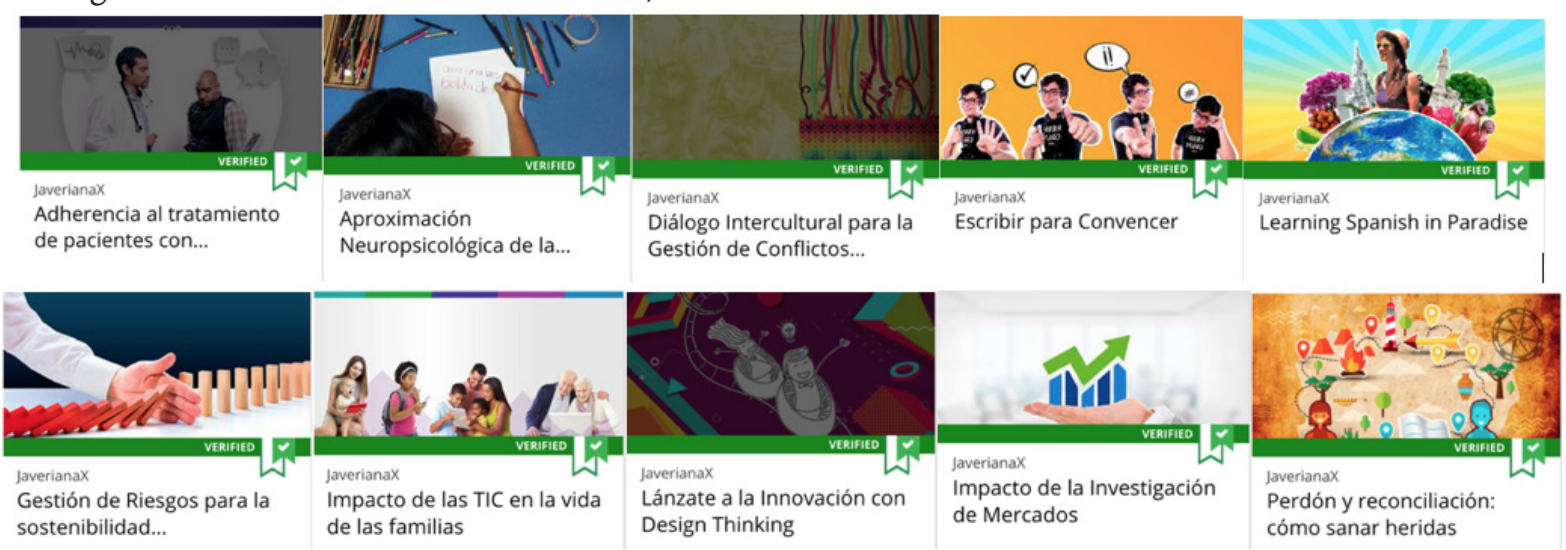

Imagen 2. Muestra de propuesta visual de los MOOCs de la Pontificia Universidad Javeriana - Cali

\section{Conclusiones y proyección del proyecto.}

La implementación de nuestros cursos ha representado un desafío, para hacer conversiones desde la alta inversión que representa crear e implementar un MOOC, hasta la participación de los usuarios en otras ofertas de la Universidad. A continuación, se proponen posibilidades de participación de públicos interno y externo en este tipo de cursos, que representen una forma efectiva de retorno a la inversión, más allá del retorno económico, en otros valores agregados para la organización. La inversión en el desarrollo de los MOOCs es una propuesta de largo aliento, pues demanda de un proceso de divulgación eficaz, y de construir cultura frente a la oferta virtual y gratuita de contenidos. En ese sentido, existen propuestas a desarrollar en el corto y mediano plazo:

- Para la divulgación de procesos de investigación y captación de nuevas propuestas que den continuidad a dichos procesos: El diseño pedagógico de los MOOC debe estar alineado con las distintas ofertas académicas de la Universidad, y permitir el reconocimiento de los créditos, la promoción institucional, de programas académicos, de grupos de investigación $\mathrm{y}$ de los investigadores, de tal forma que se identifiquen soluciones y talentos que puedan vincularse a la Universidad como generadores de nuevo conocimiento, así como escenarios de construcción colectiva y colaborativa de conocimiento que sirvan de trampolín para incentivar la publicación académica y científica derivada.

- Como propuesta pedagógica: Estos cursos deben estar articulados a una oferta académica formal, con objetivos de aprendizaje, que posibiliten el punto anterior, pero que además incursione en propuestas de innovación educativa, nuevas tendencias y estrategias, cómo la gamificación, realidad aumentada y virtual, entre otras propuestas.

- Como electiva: Los MOOCs se han usado como estrategia para diversificar la oferta de electivas de la Universidad. Como experiencia, durante los años 2017 y 2018 se contó con la apertura de 2 cursos como electiva por año, en los cuales participaron 57 y 54 estudiantes respectivamente. Por ello, se considera fundamental proyectar y diseñar cada curso como una propuesta de curso electivo para los programas académicos, lo 
que permite una sostenibilidad en el tiempo de la propuesta.

- Para generar productos derivados de Educación continua: Esta es un área en la que se han logrado intervenciones importantes, desde consultorías derivadas de los cursos, venta de productos y promoción de los servicios de la Universidad a la comunidad, y se pretende continuar generando alternativas para que las personas tengan la posibilidad de vincularse con la Universidad.

Son muchas y diversas las posibilidades que pueden surgir de la dinámica de los MOOCs. Es, en cierto modo, un campo inexplorado aún, en el qué las Universidades encontramos el camino para promover acercamientos de todos los actores sociales al mundo de la investigación y el conocimiento

\section{REFERENCIAS}

[1]. Borda, M., Maldonado, O. (2010) Estrategia nacional de apropiación social de la ciencia, la tecnología y la innovación. Bogota, Colombia: Colciencias, ISBN: 978-9588290-50-8.

[2]. Capdevila, R., \& Aranzadi, P. (2014). Los Cursos Online Masivos y Abiertos: ¿Oportunidad o Amenaza para las Universidades Iberoamericanas? RIED. Revista Iberoamericana de Educación a Distancia, volumen 17, $\mathrm{n}^{\circ} 1,69-82$.

[3]. Compañía de Jesús. (1993). Pedagogía Ignaciana: Un planteamiento Práctico. Documento Corporativo, 29-172.

[4]. Marín Agudelo, Sebastián Alejandro. Apropiación social del conocimiento: una nueva dimensión de los archivos. Revista Interamericana de Bibliotecología. 2012, vol. $35, n^{\circ} 1$; pp. 55-62.

[5]. Serna Collazos, A., Valencia Molina, C. T., Morales Rodriguez, A. (2017). MOOCS: Pontificia Universidad Javeriana-Cali: trascendiendo la gestión de contenidos, generando aprendizajes significativos. XXIII Congreso Internacional sobre Educación Bimodal TELEDU 2017.
[6]. Torres Mancera, D., \& Gago Saldaña, D. (2014). Los MOOCs y su papel en la creación de comunidades de aprendizaje y participación. RIED. Revista Iberoamericana de Educación a Distancia, 17(1).

[7]. Velazco, P. C. (2013). Relaciones entre la Pedagogía Ignaciana y las estrategias de enseñanza del modelo no directivo para el desarrollo de la conciencia emocional. Lima: Universidad Ruiz de Montoya. 